\title{
Comparación del volumen aparente de la ubre, frente a la cantidad de leche producida por Vacas Holstein Mestizas, en el cantón Chambo.
}

DOI: https://doi.org/10.33262/ap.v3i3.1.83

\begin{abstract}
(c) (1) (2)
(c)
\end{abstract}

Comparison of the apparent volume of the udder, versus the amount of milk produced by Holstein Crossbred Cows, in the canton of Chambo.

Gladys Mercedes Macas Giler. ${ }^{1}$, Fredy Bladimir Proaño Ortiz. ${ }^{2}$, Pablo Rigoberto Andino Nájera. ${ }^{3}$ \& Leidy Amarilis Alban Moreta. ${ }^{4}$

\begin{abstract}
.
Introduction. The udder (mammary system) of the cow is the most important physical asset. A large, well attached, well cared for and quality udder is very important to generate the highest milk production over a long period of time. Target. Study the relationship of the apparent volume of the udder, versus the amount of milk produced by Holstein cows. Methodology. The present study used a type of experimental research and a longitudinal method by collecting data in a given time and to determine the changes in the variables. In the present investigation, 24 crossbred Holstein females were used, 12 second and 12 third lactation. All cows were selected taking into consideration that they did not show symptoms of mastitis and their four quarters were in full production capacity. Student's $t$ test and correlation analysis by Pearson's method using SPSS version 21 were used for data tabulation. Results. The volume of the udder of the second calving Holstein cattle

\footnotetext{
${ }^{1}$ Universidad Técnica Luis Vargas Torres, Facultad de Ciencias Agropecuarias), Esmeraldas, Ecuador, gladys.giler.macas@utelvt.edu.ec ; https://orcid.org/0000-0003-1375-789.

2 Escuela Superior Politécnica de Chimborazo, Facultad de Ciencias Pecuarias. Riobamba, Ecuador. fredyproanioortiz@gmail.com: https://orcid.org/0000-0002-0937-7467.

${ }^{3}$ Escuela Superior Politécnica de Chimborazo, Facultad de Ciencias Pecuarias. Riobamba, Ecuador: pablor.andino@espoch.edu.ec: https://orcid.org/0000-0002-0515-5330.

4 Unidad Educativa Fluminense. Santo Domingo, Ecuador. leidyamarilisalbanmoreta@gmail.com: https://orcid.org/0000-0002-2479-156.
} 
was $8068.6 \mathrm{~cm}$, a value that differs significantly ( $\mathrm{P}<0.0001$ ), from the third calving cows since they registered $102497.69 \mathrm{~cm}$, while the production of milk presented highly significant differences $(\mathrm{P}>0.0001)$, due to the effect of the number of calvings, obtaining the highest value in third calving cows with 28.31 liters / milk / day and the lowest production was recorded in the second calving group with 24.03 liters / milk / day, so it is considered that increasing the number of deliveries presents a progressive increase in volume and milk production. Regarding the correlation between volume and milk production, they presented a high correlation of (0.69). Conclusion. Larger udders have a greater amount of secretory tissue and therefore a greater milk production.

Keywords: Holstein, volume, genetic correlations, milk production, lactation days, number of deliveries.

\section{Resumen}

Introducción. La ubre (sistema mamario) de la vaca es el activo físico más importante. Una ubre grande, bien adherida, bien cuidada y de calidad es muy importante para generar la mayor producción de leche en un largo período. Objetivo. Estudiar la relación del volumen aparente de la ubre, frente a la cantidad de leche producida por vacas Holstein. Metodología. El presente estudio utilizó un tipo de investigación experimental y un método longitudinal mediante la recolección de datos en un tiempo determinado y para determinar los cambios en las variables. En la presente investigación se utilizaron 24 hembras Holstein mestizas, 12 de segunda y 12 de tercera lactancia.Todas las vacas fueron seleccionadas tomando en consideración que no mostraron síntomas de mastitis y sus cuatro cuartos estuvieran en plena capacidad de producción. Para la tabulación de los datos se utilizó prueba t de student y análisis de correlación por el método de Pearson utilizando SPSS versión 21. Resultados. El volumen de la ubre de los bovinos Holstein de segundo parto fue 8068,6 cm, valor que difiere significativamente $(\mathrm{P}<0,0001)$, de las vacas de tercer parto puesto que registraron 102497,69 cm, en tanto que la producción de leche presento diferencias altamente significativas $(\mathrm{P}>0,0001)$, por efecto de número de partos, obteniéndose el mayor valor en vacas de tercer parto con 28,31 litros/leche/día y la menor producción se registró en el grupo de segundo parto con 24,03 litros/ leche/día, por lo que se considera que al incrementar el número de partos se presenta un aumento progresivo del volumen y producción de leche. Con respecto a la correlación entre volumen con la producción de leche presentaron una correlación alta de $(0,69)$. Conclusión. ubres más voluminosas tienen mayor cantidad de tejido secretor y por consiguiente una mayor producción de leche.

Palabras claves: Holstein, volumen, correlaciones genéticas, producción de leche, días de lactancia, número de partos. 


\section{Introducción.}

Ecuador representa uno de los países con más alto nivel de producción de leche en América latina y el caribe, según la literatura en el año 2016 se disponía de 896.170 vacas en ordeño con un buen nivel de producción diaria; para hablar de producción de leche es importante hacer referencia a la parte fisiológica que está involucrada en todo el proceso en este caso la ubre y no solo eso sino también tomar en cuenta diversos factores que influyen en la síntesis de la leche en el órgano ya mencionado, tales como factores endógenos de naturaleza genética, ambientales y productivos.

En lo que refiere a las características morfología externa de la ubre es uno de los factores con influencia dentro de los estudios de genética y selección, sin embargo, estos varían por lo que se ve reflejado en la producción, considerando entonces que aquellas más voluminosas son las que tendrán mayor producción, a partir de esta referencia se puede establecer la capacidad entre una y otra. Con el objetivo de proporcionar una guía para el manejo ganadero se realiza este estudio que busca establecer una relación entre el volumen aparente de la ubre en vacas Holstein mestizas con la producción de leche.

\section{Metodologia}

La investigación se desarrolló en las siguientes haciendas ganaderas de la provincia de Chimborazo: San Jorge de Balcashilocalizada en el cantón Riobamba en la parroquia Quimiag Comunidad de Balcashi a unos $15 \mathrm{~km}$ al este de Riobamba; hacienda Rocón se encuentra ubicada al sureste del cantón Chambo a una distancia de $5 \mathrm{~km}$ desde el parque central de Chambo, en la parroquia San Miguel Guaructús y hacienda Pucate ubicada en el cantón Chambo parroquia Chambo, al sureste del Cantóna $1 \mathrm{~km}$ de la cabecera cantonal.

\section{Unidades experimentales}

En la presente investigación se utilizaron 24 hembras Holstein mestizas, 12 de segunda y 12 de tercera lactancia.Todas las vacasfueron seleccionadas tomando en consideración que no mostraron síntomas de mastitis y sus cuatro cuartos estuvieran en plena capacidad de producción.

Tipo de investigación

El presente estudio utilizó un tipo de investigación experimental que consistió en la manipulación de las variables moformétricas de la ubre y su efecto durante el número de partos; a su vez se aplicó el análisis de correlación por el método de Pearson utilizando INFOSTAT versión 2017.

Método de investigación

La presente investigación utilizó un método longitudinal, mediante la recolección de datos en un tiempo determinado y para determinar los cambios en las variables. 
Tratamientos y diseño experimental

Se utilizaron 24 Hembras bovinas, divididas en dos tratamientos: T1=vacas de segundo parto; $\mathrm{T} 2=$ vacas de tercer parto. Cada tratamiento estuvo conformado por 12 vacas, cada una de las cuales constituyó una unidad experimental. Las mismas que se distribuyeron bajo un Diseño Completamente al Azar correspondientes al siguiente modelo lineal.

$Y \boldsymbol{i j}=\mathrm{X}+\boldsymbol{T} \boldsymbol{i}+\boldsymbol{E} \boldsymbol{i j}$

Dónde:

$\boldsymbol{Y} \boldsymbol{i} \boldsymbol{j}$ : Valor de la variable en determinación.

$\mathrm{X}$ : Valor de la media general.

$\boldsymbol{T} \boldsymbol{i}:$ Efecto de los tratamientos

$\boldsymbol{E} \boldsymbol{i j}$ : Efecto del error experimental

En la tabla 1, se describe el esquema del experimento empleado.

Tabla 1. Esquema del experimento

\begin{tabular}{lcccc}
\hline \# de Parto & Código & Rep. & TUE & $\begin{array}{c}\text { Total de } \\
\text { animales }\end{array}$ \\
\hline Segundo. & T2 & 12 & 1 & 12 \\
Tercer. & T2 & 12 & 1 & 12 \\
Total de animales & & & & 24 \\
\hline
\end{tabular}

Fuente: Elaboración propia.

Mediciones experimentales.

Para la toma de las medidas descritas, se siguió el procedimiento propuesto por Espinosa, Y, (2013), que consistió en relacionar el volumen mediante el cálculo de la profundidad, longitud y ancho de la ubre, según la ecuación: $\mathrm{V}=(\mathrm{A} * \mathrm{P} / 6) *[(3 * \mathrm{~L} * \mathrm{~A}+(\mathrm{P} 2)]$; donde:

$\mathrm{V}=$ Volumen aparente $(\mathrm{cm} 3)$.

$\mathrm{A}=$ Ancho de la ubre $(\mathrm{cm})$.

$\mathrm{P}=$ Profundidad $(\mathrm{cm})$.

$\mathrm{L}=$ Longitud (cm).

- Los registros de medidas de las ubres se llevaron a cabo en el ordeño de la madrugada 4: 30 am y en el de la tarde 15:45 pm

- Para el cálculo del volumen se tomó como referencia lo descrito por Espinosa estableciendo entonces una relación entre la profundidad, longitud y ancho de la ubre según la ecuación antes indicada. 
Para tomar las medidas de profundidad, longitud, ancho de la ubre, SPA, SPP, SPAP, se utilizó una regla $\mathrm{T}$ de $60 \mathrm{am}$, una regla zoometría, una regla de $30 \mathrm{~cm}$, cinta métrica de $100 \mathrm{~cm}$, respectivamente.

Los resultados experimentales se determinaron mediante los siguientes procesos estadísticos: Prueba de hipótesis para variables continuas segúnt de student al $\alpha \leq 0.05$.

Se empleó el análisis de regresión para analizar la relación entrelos días de la lactancia entre las variables volumen / producción de leche. Análisis de correlación de Pearson para determinar la relación presente entre las variables estudiadas. Los datos de la relación del volumen aparente de la ubre con la producciónde leche se determinaron mediante el paquete estadístico SPS (versión 21).

\section{Resultados.}

El ancho de las ubres de las vacas utilizadas en el presente estudio, fueron altamente significativas $(\mathrm{P} \geq 0,0001)$ entre el segundo y tercer parto, con medidas de 18,79 $\pm 0,15 \mathrm{y}$ $19,64 \pm 0,15 \mathrm{~cm}$ respectivamente.

El promedio del ancho de las ubres fue similar al indicado por Muñoz (2017), quien reportó un promedio de 19,19 cm, medición que reportó al investigar vacas Holstein mestizas bajo condiciones medio ambientales similares a las de la presente investigación; este autor menciona que, las probables diferencias en el ancho de las ubres de vacas Holstein, se podrían ser causa de la madurez y del estado funcional de las vacas, así como a las características individuales y raciales de cada animal.En esto último, Rizzi (2007), obtuvo un promedio de $15,67 \mathrm{~cm}$ en vacas Caroras, por tanto esta investigación también coincide en el hecho de que el anchode la ubre de las vacas es una medida que cambia en función de la raza y genética, en el mismo contexto mencionado Casanova et al (2012), evaluaron la aptitud de la ubre donde reportaron una media de $23.3 \mathrm{~cm}$ en consideración a los días de lactancia, por su parte Espinoza et al en el año (2013), muestra estudios en búfalas con un promedio donde se reporta valores de $21,54 \mathrm{~cm}$, lo que corrobora la importancia que representa el ancho de la ubre como parámetro primordial y de relevancia al momento de evaluar la capacidad de la ubre.

Profundidad, $\mathrm{cm}$.

Para la variable profundidad de las ubres se observarón diferencias estadísticas altamente significativas $(\mathrm{P} \geq 0.0001)$, donde las vacas Holstein mestizas de segundo parto presentaron $35,32 \pm 0,14 \mathrm{~cm}$ y las de tercer parto $(40,01 \pm 0,14 \mathrm{~cm})$, por lo que se puede deduir que al incrementar el número de partos en las vacas Holstein se incrementa la profundidad. El prometido registrado entre los grupos de animales fue de $37,66 \mathrm{~cm}$, ante este resultado no se reportan investigaciones en bovinos lecheros donde se haya utilizado una metodología similar para medir la profundidad. Sin embargo, Espinoza, et al (2013), al realizar la evaluación entre la morfometría de la ubre y producción de leche en búfalas, registro un ancho de $25,30 \mathrm{~cm}$, las diferencia reportadas puede deberse a que el investigador realizó el cálculo en búfalas animales que presentan una genètica y 
conformación en las ubres difirentes en comparación con los bovinos. Por su parte Rizzi (2007), al considerar la profundidad como la distancia desde el piso a la ubre, reporta valores de $28,92 \mathrm{~cm}$.

En relación a los datos registrados en la presente evaluación la Federación de Holstein Friesan(2004), asocian la profundidad con cisternas grandes, sin embrago también están más predispuestas a lesiones y a la aparición de mamitis. Además, es importante manifestar que la profundidad de la ubre se encuentra íntimamente relacionada con la altura del animal; lo que es corroborado por Estrella (2015), quien menciona que para determinar la capacidad de las ubres en las vacas Holstein es necesario tomar en cuenta el número de lactancias, debido a que ubres que tienen una mayor profundidad son más sensibles a lesiones y por ende a contacto con agente patógenos como los gérmenes, por lo antes indicado se prefiere una ubre de profundidad moderada que no gènere complicacions en el animal.

Longitud, $\mathrm{cm}$.

Para la longitud presente en vacas Holstein mestizas se observarón diferencias altamente significativas $(\mathrm{P} \geq 0,0019)$, en relación al número de partos, con 55,08 $\pm 0,15 \mathrm{~cm}$ para vacas de segundo parto y 55,76 $\pm 0,15 \mathrm{~cm}$ para vacas de tercer parto, en función a estos resultados no se reportan investigaciones en bovinos lecheros que permitan inferir con la longitud de las ubres encontrada en las vacas Holstein mestizas.

Sin embargo, se observan investigaciones efectuadas en búfalas donde Espinoza, et al (2013) donde se registró una longitud de $38,46 \mathrm{~cm}$, valores inferiores a los reportados en la presente investigación, diferencias que se deben a que el investigador realizó su reporte en búfalas, el presente autor estableció en función a sus resultados que el tamaño de la longitud está en dependencia al desplazamiento que puede tener la ubre hacia la parte craneal que beneficia a las ubres.

Volumen, $\mathrm{cm} 3$.

Como se indicó anteriormente, para el cálculo del volumen aparente de las ubres, se consideraron las mediciones de ancho, y profundidad y longitud. Las diferencias estadísticas $(\mathrm{P} \geq 0,0001)$ de todas las medidas citadas anteriormente en los resultados mantuvieron las mismas tendencias estadísticas, resultados que también se ve reflejado en el cálculo del volumen aparente, reportando diferencias altamente significativas ( $\mathrm{P}$ $\geq 0,0001$ ), entre el segundo y tercer parto, con medidas de 80686,23 $\pm 575,88$ y 102497,69 $\pm 575,88 \mathrm{~cm} 3$ ), respectivamente, por lo que se considera que al incrementar el número de partos las vacas presentan un aumento progresivo del volumen.

El promedio del volumen de las ubres de las vacas de segundo parto y del tercer parto resultó de 91591,96 cm3 los resultados registrados difieren de Espinoza, et al (2013), quien realizó la evaluación entre la morfometria de la ubre y producción de leche en búfalas, investigador que obtuvo un volumen de 42536,7 cm3, esta diferencia se debe a que el investigador realizó su estudio en búfalas. 
Por su parte Ayadi, en el año (2003), menciona que las características morfológicas que propician el desarrollo glandular están en función del ancho, profundidad y longitud de las ubres.

Producción de leche, L.

La producción de leche registró diferencias estadísticas altamente significativas $(\mathrm{P} \geq 0.0001)$, por efecto de número de partos, con 24,03 $\pm 0,32$ litros/ leche/día para vacas de segundo parto y $28,31 \pm 0,32$ litros/leche/día para vacas de tercer parto.

El promedio de producción de leche en la presente investigación fue de 26, 17 litros/día valor que difiere de los obtenidos por García (2001) y Muñoz (2017), con un promedio de 22,02 y 18,68 litros /día respectivamente; investigadores que consideraron que, probables diferencias se deben principalmente a la mejora constante de la genética de los animales.

Por su parte, Olivera, (2001), al determinar los índices de producción láctea en vacas de primer, segundo y tercer parto, presentó un promedio de 20 litros/día demostrando un incremento en la producción de láctea a medida que incrementaba el número de partos; los resultados obtenidos en la investigación le permitieron concluir que una buena ubre, es la que cuando está llena, es voluminosa y profunda, elástica, consistente y suave al tacto.

Las diferencias encontradas entre las investigaciones talvez se deben a lo indicado por Luz, (2014), donde indica que las glándulas mamarias constantemente presentan procesos de crecimiento durante todo el estado de preñez, ocurriendo la proliferación y ramificación de los canales lactíferos, túbulos y alvéolos. La ubre presenta un continuo desarrollo en relación de las cantidades y tamaño de las células durante toda la primera lactancia hasta la quinta lactancia.

Casanovas, et al, (2012), al investigar la fisiología de la lactancia también indicaron que la ubre debe presentar una estructura extensa, con un gran potencial de producción y almacenamiento diario de leche que está en relación a la cantidad de tejido secretor, a la vez también señalan que deben presentar buenos ligamentos laterales para una buena inserción en el abdomen y un fuerte ligamento medio, el cual servirá de apoyo primordial para la producción diaría de leche; bibliografía que corroborado por Luz, (2018), quien menciona que la cantidad de tejido secretor y células secretoras son componentes de gran importancia y limitantes en la capacidad de producción de la ubre. En relación a los autores se puede indicar que una ubre de apariencia grande puede aparentar una gran cantidad de leche, pero también pueden presentar una mayor proporción de tejidos conectivos y menor cantidad de parénquima glandular, el cual representa las células productoras y encargadas de la secreción de leche, en conclusión, ubres más profundad que no sobrepase los corvejones determinarán una producción eficiente de leche.

Correlación entre las variables morfológicas de la ubre. 
El Volumen aparente de la ubre comparada con la producción de leche, registró una correlación alta positiva de 0,69 (Tabla 2); este resultado difiere del indicado por Espinoza etal (2013), con una valor de 0,49, el mismo que se lo puede considerar como equivalente a una correlación media; las diferencia reportadas puede deberse a que el investigador realizó el cálculo en búfalas y sus resultados le permitieron establecer que las ubres más voluminosas son las que producen más producción de leche tienen, afirmación que respaldan varios autores (Ramella et al, 2003; Linzell, 2008; y Luz, I, (2014), en estudios diferentes.

Tabla 2:Valores de correlaciones altas y medias entre las variables morfométricas.

\begin{tabular}{cccc}
\hline Correlación & Valor & $\begin{array}{c}\text { Nivel de } \\
\text { confianza }\end{array}$ & Nivel \\
\hline Volumen vs producción de leche & 0,69 & 0,001 & Alto \\
SPA vs SPP & 0,78 & 0,001 & Alto \\
SPDr vs SPIz & 0,83 & 0,001 & Alto \\
LPT vs LPD & 0,70 & 0,001 & Alto \\
Ancho vs Longitud & 0,33 & 0,001 & Medio
\end{tabular}

SPA: Separación de los pezones anteriores/ SPP: Separación de los pezones posteriores / SPDr: Separación entre los pezones derechos/SPIz: Separación entre los pezones izquierdo / LPD: Largo de los pezones delanteros / LPT: Largo de los pezones traseros /DPD: Diámetro de los pezones delanteros / DPT: Diámetro de los pezones traseros.

\section{Fuente: Elaboración propia.}

Al relacionar la separación de los pezones anteriores con los posteriores registró una correlación alta positiva de 0,78(Tabla 2),resultado positivo según Duran (2012), quien menciona que la colocación y nacimiento de los pezones no estar muy cerrados en los cuartos anteriores yposteriores de la ubre para realizar un ordeño eficiente, además un correcta posición de los pezones es importante y esencial para efectuar un ordeño correcto que disminuyan las lesiones que puedan presentarse durante el ordeño.

La separación entre los pezones derechos comparada con los izquierdos registró una correlación alta de 0,83 (Tabla 2), estos resultados muestran un balance positivo en la ubre que permitan un ordeño de formas más rápida y completamente de las vacas. Por su parte Sharma et al, en el año (2016), mencionaron que una distancia correcta y balanceada del pezón es de vital importancia para un ordeño eficiente, ya que es prevendría la caída de la máquina ordeñadora y posible contaminación bacteriana.

La correlación entre el largo de los pezones delanteros con los pezones traseros presentó una correlación alta positiva de 0,70 (Tabla 2), resultados que señalan un mejoramiento entre estas características beneficia en el proceso de ordeño. Lo que es corroborado por Peñafiel (2017), quien señala que la presencia de pezones muy cortos no puede ser colocados de forma correcta en las pezoneras pudiendo generar daños a las ubres y consecuentemente la disminuir la vida productiva de las vacas. 
Al relacionar el ancho de la ubre con la longitud de la ubre presentó una correlación media positiva de 0,33 (Tabla 2), valor que difiere de los señalados por Bhuiyan, et al (2004), con 0,78 ; el mismo que se lo puede establecer como una correlación alta y positiva, estos resultados obtenidos por el autor le permitieron concluir que a un mayor ancho de la ubre genera una ubre amplia y presenta un efecto positivo y directo sobre cantidad y capacidad de almacenamiento de la glándula, afirmación que es corroborada por Arias et al (2003).

\section{Conclusiones.}

- Se ratifica que el volumen aparente de las ubres, tienen relación con el número de partos, aligual que la producción de leche.

- Se encontró que, a mayor volumen aparente de las ubres, los niveles de producción de lechetambién se incrementan.

- La separación de los pezones anteriores progresa de forma simétrica con la separación delos pezones posteriores, al igual que el largo de los pezones delanteros y traseros.

- Se encontró que ubres con un mayor ancho posterior condicionan una mayor longitud en eltamaño de la ubre.

- Incrementos en el diámetro de los pezones delanteros y trasero reducen la separación entrepezones anteriores y posteriores al igual que los derechos e izquierdos.

\section{Referencias bibliográficas.}

Arias , D. Hernández, M y Torres, L.(2003).La ubre puede definir el sistema de alimentación: "Desarrollo territorial sustentable de la zona mixta de la Provincia de Santa Fe, Argentina".'Recuperado:https://inta.gob.ar/sites/default/files/script-tmpinta_-_cruzamiento_con_jersey_bretschneider_g_2014.pdf.

Ayadi, M. (2003). Estructura de la ubre y la frecuencia de ordeño en vacas lecheras.( Tesis de Doctorado, Universidad Autónoma de Barcelona).Recuperado:https://ddd.uab.cat/pub/tesis/2003/tdx-0621104151003/ma1de2.pdf Pp25-28.

Bhuiyan, M. M. (2004). Importance of Mammary System Conformation Traits in Selecting Dairy Cows on Milk Yield in Bangladesh MM Bhuiyan, MR Islam, ML Ali, MK Hossain," MA Kadir, NS Lucky and BR Das. Journal of Biological Sciences, 4(2), 100-102.

Casanovas, E, Muñoz, L y Chávez, A. (2012). Evaluación de la capacidad de la ubre en el ordeño mecánico en vacas lecheras. (Tesis de maestría. Universidad de Cienfuegos).

Recuperado:

file:///C:/Users/DELL/Downloads/Evaluacion\%20zootecnica\%20de\%20la 
$\% 20$ aptitud\%20de\%20la\%20ubre\%20para\%20el\%20ordenno\%20mecanic o\%20en\%20vacas\%20lecheras\%20(2).pdf- PP 7-8.

Duran, J, (2012). Análisis de correlación y regresión entre los caracteres fenotípicos del tipo lechero, con la producción lechera alcanzada, de vacas HolsteinFriesian, en la cuenca lechera de Machachi. (Tesis de pregrado, Universidad Central del Ecuador). Recuperado: http://biblioteca.udenar.edu.co:8085/atenea/biblioteca/90671.pdf.

Espinosa-Núñez, Y., Capdevila-Valera, J., Ponce-Ceballos, P., Riera-Nieves, M., \& Nieves-Crespo, L. (2013). Relación entre morfología de la ubre y la producción y composición de la leche en búfalas. Revista Científica, 23(3), 220-225.

Estrella, F. (2015). Evaluación del hato lechero de la Estación Experimental Tunshi, utilizando el programa de cruzamiento ganadero SelectMatingService (sms). (Tesis de pregrado,Escuela Superior Politécnica de Chimborazo). Recuperado: http://dspace.espoch.edu.ec/bitstream/123456789/5270/1/tesis\%20compl eta\%20fabian.pdf.

Luz, I. (2014). Sistema mamario. Recuperado: https://ganaderiasos.files.wordpress.com/2014/07/sistema-mamario-esp.pdf PP 4-5.

Muñoz, G. (2017). Evaluación bovinométrica y productiva del rejo en el 12 programa bovinos de leche Tunshi. (Tesis de pregrado, Escuela Superior Politécnica de Chimborazo).

Recuperado:http://dspace.espoch.edu.ec/bitstream/123456789/7755/1/17 T1493.pdf.

Olivera, S. (2001). Índices de producción y su repercusión económica para un establo lechero. Revista de Investigaciones Veterinarias del Perú, 12(2), 49-54.

Peñafiel, R. (2017). Evaluación del hato lechero del centro de excelencia agropecuario de Bucay, utilizando el programa de cruzamiento ganadero selectmatingservice. (Tesis depregrado, Escuela Superior Politécnica de Chimborazo). Recuperado: http://dspace.espoch.edu.ec/bitstream/123456789/7096/1/17T1466.pdf.

Rizzi, et al (2007), Parámetros genéticos de las características morfológicas de ganadoCarora. Recuperado: http://ve.scielo.org/scielo.php?script=sci_arttext\&pid=S079822592007000100009. 
Sharma, A., Sharma, S., Singh, N., Sharma, V., \& Pal, R. S. (2016). Impact of udder and teat morphometry on udder health in Tharparkar cows under climatic condition of hot arid region of Thar Desert. Tropical animal health and production, 48(8), 1647-1652.

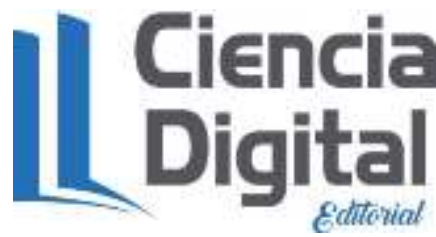




\section{PARA CITAR EL ARTÍCULO INDEXADO.}

Macas Giler, G. M., Proaño Ortiz, F. B., Andino Nájera, P. R., \& Alban Moreta, L. A. (2021). Comparación del volumen aparente de la ubre, frente a la cantidad de leche producida por Vacas Holstein Mestizas, en el cantón Chambo . AlfaPublicaciones, 3(3.1), 140-151. https://doi.org/10.33262/ap.v3i3.1.83

\section{LCiencia}

El artículo que se publica es de exclusiva responsabilidad de los autores y no necesariamente reflejan el pensamiento de la Revista Alfa Publicaciones.

El artículo queda en propiedad de la revista y, por tanto, su publicación parcial y/o total en otro medio tiene que ser autorizado por el director de la Revista Alfa Publicaciones.
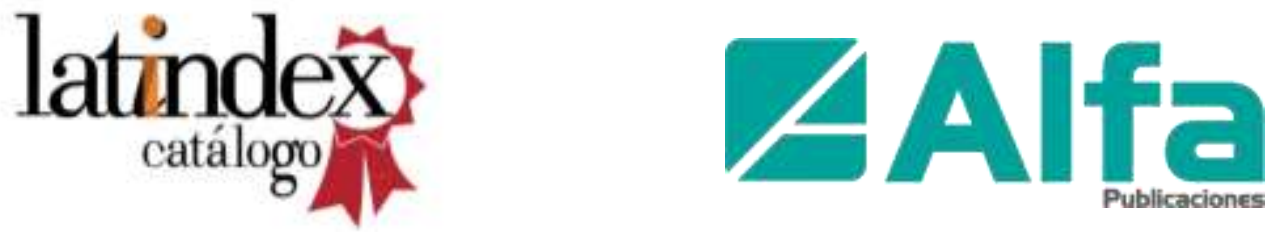